\title{
Changes in clinical disease activity are weakly linked to changes in MRI inflammation on treat-to-target escalation of therapy in rheumatoid arthritis
}

Fiona M. McQueen ${ }^{1,5^{*}}$, Peter Chapman ${ }^{6}$, Terina Pollock ${ }^{2}$, Dena D'Souza ${ }^{1}$, Arier C. Lee ${ }^{3}$, Nicola Dalbeth ${ }^{4,5}$, Lisa Stamp ${ }^{7}$, Karen Lindsay ${ }^{5}$ and Anthony Doyle ${ }^{2}$

\begin{abstract}
Background: Rheumatoid arthritis (RA) treat-to-target (T2T) regimens often use the disease activity score (28 joints) incorporating C-reactive protein (DAS28 $8_{\text {CRP }}$ ) as an outcome measure. We compared changes in the DAS28 $8_{\text {CRP }}$ with changes in magnetic resonance imaging (MRI) inflammation on treatment escalation.

Methods: Eighty seropositive RA patients with active disease were enrolled. Group A ( $N=57)$ escalated to another conventional disease-modifying therapy (CDMARD) combination, and Group B $(N=23)$ to anti-TNF therapy/ CDMARDs. Contrast-enhanced 3T-MRI wrist scans were obtained before and 4 months after regimen change. Scan pairs were scored for inflammation (MRI(i)) and damage. Disease activity was assessed using the DAS28CRP.

Results: Eighty patients were enrolled and $66 \mathrm{MRI}$ scan pairs were available for analysis. Intra-reader reliability was high: intraclass correlation coefficient (average) 0.89 (0.56-0.97). $\triangle \mathrm{DAS} 28_{\mathrm{CRP}}$ did not differ between groups: Group A, $-0.94(-3.30,1.61)$; Group B, $-1.53(-3.59,0.56)(p=0.45)$. $\Delta$ MRI(i) also did not differ: Group A, $0(-25,10)$; Group B, $-1(-15,28)(p=0.12)$. Combining groups, $\Delta M R I(i)$ correlated weakly with $\triangle$ DAS28 CRP (Spearman's $0.36, p=0.003$ ). Using multiple linear regression analysis adjusting for confounders, $\triangle \mathrm{DAS} 28_{\mathrm{CRP}}$ was associated with $\Delta \mathrm{MRI}(\mathrm{i})(p=0$. 056). Of the individual MRI measures, only $\Delta$ tenosynovitis correlated with $\triangle \mathrm{DAS28}$ CRP (Spearman's $0.33, p=0.007$ ). $\Delta \mathrm{MRI}(\mathrm{i})$ was negatively associated with the MRI erosion score at entry $(p=0.0052)$.

Conclusions: We report the first study investigating the link between changes in clinical and imaging inflammation in a real-world RA cohort escalating to conventional and biologic DMARDs. The association was significant but relatively weak, suggesting that MRI targets cannot yet be advocated as outcomes for T2T escalation.
\end{abstract}

Trial registration: ANZCTR 12614000895684. Registered 22 August 2014.

\section{Background}

Rheumatologists routinely manage rheumatoid arthritis (RA) using a treat-to-target (T2T) regimens [1], escalating therapy in patients who fail to reach remission or a low disease activity state [2]. Management is often initiated with methotrexate alone and then escalation proceeds to

\footnotetext{
* Correspondence: f.mcqueen@auckland.ac.nz

'Department of Molecular Medicine and Pathology, Faculty of Medical and Health Sciences, The University of Auckland, Private Bag 92019, Auckland, New Zealand

${ }^{5}$ Department of Rheumatology, Greenlane Clinical Centre, Auckland District Health Board, 214 Green Lane West, Epsom, Auckland 1051, New Zealand

Full list of author information is available at the end of the article
}

combination therapy with conventional disease-modifying anti-rheumatic agents (cDMARDs) and biologic DMARDs (bDMARDs). In New Zealand, cDMARD combinations include triple therapy (methotrexate, sulphasalazine, hydroxychloroquine) and methotrexate/leflunomide, although other options are available. The New Zealand Pharmaceutical Management Agency (PHARMAC), responsible for buying and funding pharmaceuticals, has defined criteria for funding bDMARDs including a trial of three different cDMARD combinations [3]. For those with an inadequate response to cDMARDs, escalation to methotrexate/antitumour necrosis factor (anti-TNF) agents follows and 
subsequently other bDMARDs. Adequacy of response is usually measured by the DAS $28_{\text {CRP }}[4,5]$.

MRI is a sensitive imaging modality that can reveal articular inflammation and joint damage [6, 7]. The OMERACT RA MRI score (RAMRIS) [8] and the Harvaardsholm tenosynovitis score [9] can quantify inflammation in a relatively reproducible manner. Studies show that MRI can detect more inflammation than physical examination. Up to $96 \%$ of asymptomatic RA patients with normal joints have MRI synovitis and 46\% have MRI bone oedema/osteitis [10]. Krabben et al. [11] found MRI inflammation in $66 \%$ of non-swollen wrist joints in patients with early RA. Severe grades of bone oedema (osteitis) were common in non-swollen joints. We and others have found that osteitis is a predictor of joint erosion [12, 13]. Although MRI is more sensitive than clinical assessment for detection of joint inflammation and damage, MRI outcomes are not yet part of accepted clinical practice because of feasibility issues including cost and accessibility. It is also not clear whether using the change in MRI inflammation scores to dictate escalation of therapy would lead to better outcomes than using easily administered clinical scores such as the DAS28 $8_{\text {CRP. }}$. While clinical trials of bDMARDs including golimumab [14] and tocilizumab [15] indicated that clinical responses were associated with improvements in MRI inflammation scores, there is little information comparing clinical and MRI responses to cDMARDs in the T2T "real-world" setting.

The aim of this study was to compare the response in clinical disease activity (DAS28 $8_{\mathrm{CRP}}$ ) with response in MRI inflammation scores after T2T-dictated escalation of therapy in two groups of RA patients: those escalating from cDMARDs to a different cDMARD combination and those escalating to a combination that includes anti-TNF therapy.

\section{Methods}

\section{Power calculation}

Studies exploring the relation between $\triangle \mathrm{MRI}$ synovitis scores and $\triangle \mathrm{DAS} 28_{\mathrm{CRP}}[7,16,17]$ indicated a simple correlation of 0.4 . Using a two-sided test at the $5 \%$ significance level with $80 \%$ power, the required total sample size would be 46 patients. Allowing for 10\% dropout, the estimated sample size required was 50 . For comparing MRI(i) responses between patients escalating to cDMARDs versus cDMARD/anti-TNFs, sample size was calculated based on the one-sided hypothesis that triple therapy would not be inferior to MTX/anti-TNF. We estimated that with 50 patients per group the study would have $80 \%$ power at 5\% significance to establish non-inferiority, assuming a between-group difference in change from baseline to 3 months of $2(S D=2)$. Although enrolment was continued for 3 years, only 80 patients with seropositive RA were recruited from rheumatology outpatients' clinics from Auckland and Christchurch, New Zealand between
2014 and 2016. Two cohorts were recruited: Group A included patients on CDMARD therapy (either methotrexate alone or a cDMARD combination), escalating to a different cDMARD combination; and Group B included patients on a cDMARD combination who were escalating to bDMARDs including an anti-TNF agent. Patients were required to have a DAS28 $8_{\mathrm{CRP}} \geq 3.2$ at the first clinical visit. Tender joint counts (28 and 68 joints) and swollen joint counts (28 and 66 joints) were performed by trained research nurses and patients filled out questionnaires to assess function. Serum was obtained for inflammatory markers and rheumatoid serology. Written informed consent was obtained from all patients. The study was approved by the Health and Disability Ethics Committees, New Zealand.

\section{MRI scans and scoring}

The dominant wrist was scanned using a 3 T scanner (Philips MR Systems Achieve $3 \mathrm{~T}$; Koninklijke Philips Electronics NV, Eindhoven, the Netherlands). An eightelement Philips SENSE 3.0 T Wrist Coil 8 Channel (Invivo Corp, Gainsville, FL, USA) (receive only) was used. The dominant hand in the wrist coil fitted snugly by the patient' s side, palm to body, thumb anteriorly. The field of view included the carpus, distal radioulnar joint (dRUJ) and metacarpal (MC) bases. The following were used: turbo spin echo sequences, T1 weighted (T1w) and T2 weighted (T2w) with fat saturation (FS) using spectral adiabatic inversion recovery (SPAIR) in axial and coronal planes and proton density (PD) coronals (without FS). T1wFS axial and coronal sequences were obtained post intravenous gadolinium diethylenetriamine pentaacetic acid (GdDTPA) at standard dosage (Omniscan (Gadodiamide; $5.0 \mathrm{mmol} / 10 \mathrm{ml}$ or $2.87 \mathrm{~g} / 10 \mathrm{ml}$ ); GE Healthcare, Inc., Princeton, NJ, USA). Synovitis, osteitis and erosions were scored according to RAMRIS [8] and tenosynovitis in the flexor and extensor compartments [9]. MRI scan pairs (baseline, 4 months) were scored in known chronological order by one experienced reader (AD), blinded to clinical and X-ray data. Ten scans were selected randomly and rescored 6 months later. The intra-reader reliability was high: ICC (av) = 0.89 (99\% CI 0.56-0.97).

\section{X-rays}

Plain radiographs of the hands and feet were obtained for all patients on enrolment. They were scored for damage using the Sharp van der Heijde $(\mathrm{SvdH})$ score [18] by a rheumatologist (ND), blinded to clinical and MRI data.

\section{Statistical analysis}

Intra-reader reliability for MRI scores was determined using intraclass correlation coefficients (ICCs). Spearman's correlations were used to examine the association between $\triangle \mathrm{DAS} 28_{\mathrm{CRP}}$ and $\triangle \mathrm{MRI}$ scores. Clinical characteristics for 
Groups A and B were compared using the chi-square test and median two-sample test. The effect of $\triangle \mathrm{DAS} 28_{\mathrm{CRP}}$ and treatment group on $\triangle \mathrm{MRI}(\mathrm{i})$ were analysed using multiple linear regression adjusting for age, gender, duration, anti-CCP titre and MRI erosion score at baseline. Four patients transited from Group A into Group B, providing eight scan pairs. Apart from analyses investigating baseline patient characteristics where the four patients were included only in their initial Group A, all analyses were performed including these patients in both groups and were conducted using SAS for Windows version 9.4 (SAS Institute Inc., Cary, NC, USA).

\section{Results}

A total of eighty patients were enrolled, fifty-seven in Group A (42 female, 15 male) and 23 patients in Group B (5 female, 18 male) $[5,18]$. Dropout throughout the protocol is summarized in Fig. 1. One patient began adalimumab for disease control 1 month after enrolment and remained on this bDMARD for 3 months before the second MRI scan and so was switched to Group B. Full clinical and contrast-enhanced MRI data from Visit 1 (baseline) and Visit 2 (4 months) were available for 62 patients. However, 66 scan pairs were available for analysis (including eight pairs from four patients who were sequentially enrolled into Group A then Group B).

\section{Clinical characteristics}

Patients in Group A and Group B were of similar age and gender but those in Group B had a longer disease duration (Table 1). Consistent with their different places on the T2T escalation ladder, swollen and tender joint counts and disease duration (Visit 1) were lower in Group A than Group B (Table 2). The DAS28 $8_{\text {CRP }}$ was also lower for Group A than Group B: 4.24 vs $5.15(p=0.007)$. Interestingly, there was no difference between groups for MRI synovitis or tenosynovitis scores at Visit 1 but total MRI inflammation and osteitis scores were lower in Group A than Group B ( $p$ $=0.05$ and 0.02 respectively) (Table 2). MRI erosion scores were also lower in Group A than Group B: 4 (0-50) and 9 (1-47) respectively $(p=0.03)$. The total SvdH X-ray damage score (Visit 1) was also lower in Group A than Group B: $20.5(0-217)$ and $63(0-209)$ respectively $(p=0.03)$.

\section{Changes in clinical disease activity and MRI inflammation on escalation}

Patients' responses were measured by $\triangle \mathrm{DAS} 28_{\mathrm{CRP}}$. Both groups improved from Visit 1 to Visit 2 so that $\triangle \mathrm{DAS} 28_{\mathrm{CRP}}$ for Group A was $-0.94(-3.30,1.61)$ and for Group B was $-1.53(-3.59,0.56)$. There was no difference between groups $(p=0.45)$. For Groups A and B, $83.3 \%$ and $87.5 \%$ respectively had a fall in the DAS28 $8_{\text {CRP }}$ from Visit 1 to Visit 2 indicating clinical improvement, while in $16.7 \%$ and $12.5 \%$ there was worsening. $\triangle \mathrm{MRI}(\mathrm{i})$ from Visit 1 to Visit 2 varied between patients but median scores showed little change over 4 months, despite escalation of therapy, and there was no difference between groups. For Group A, $\triangle \mathrm{MRI}(\mathrm{i})$ median (range) was $0(-25,10)$; for Group B, $-1(-15,28)$ $(p=0.12)$. Table 3 shows how patients responded in terms of MRI inflammation (total and sub-scores) and MRI erosion score. Only a few showed MRI erosion progression (three patients (6.98\%) in Group A and five patients

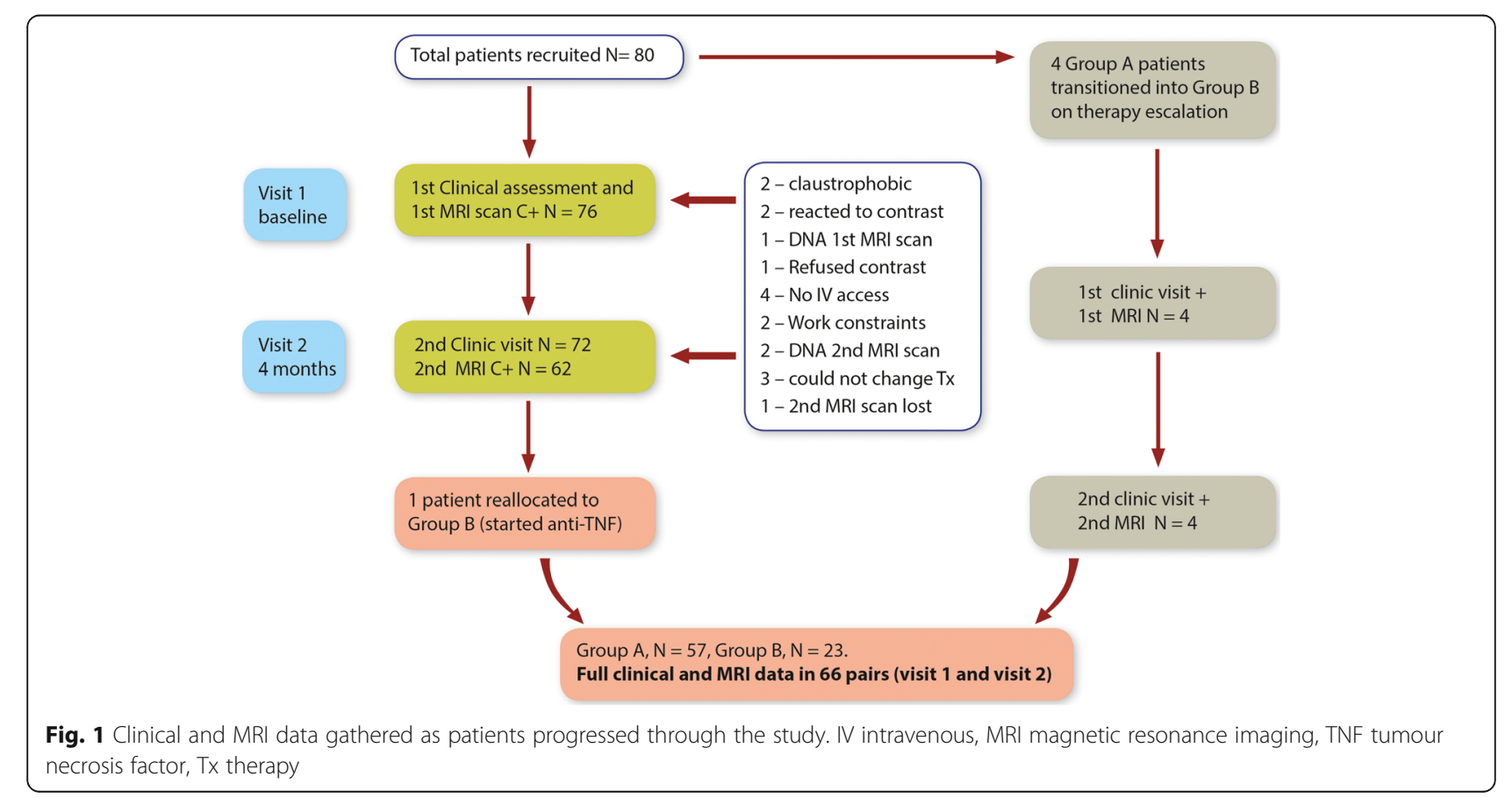


Table 1 Patient characteristics on enrolment

\begin{tabular}{|c|c|c|c|c|c|c|c|}
\hline \multirow{2}{*}{$\begin{array}{l}\text { Clinical characteristic at } \\
\text { recruitment }\end{array}$} & \multicolumn{2}{|c|}{ Total } & \multicolumn{2}{|c|}{ Group A } & \multicolumn{2}{|c|}{ Group B } & \multirow{2}{*}{$\begin{array}{l}\text { Chi-square } \\
p \text { value* }\end{array}$} \\
\hline & \multicolumn{2}{|c|}{$(N=80)$} & \multicolumn{2}{|c|}{$(N=57)$} & \multicolumn{2}{|c|}{$(N=23)$} & \\
\hline \multicolumn{8}{|l|}{ Gender } \\
\hline Female & 60 & $75 \%$ & 42 & $73.68 \%$ & 18 & $78.26 \%$ & 0.67 \\
\hline Male & 20 & $25 \%$ & 15 & $26.32 \%$ & 5 & $21.74 \%$ & \\
\hline Age (months) & \multicolumn{2}{|c|}{$54.5(24,82)$} & \multicolumn{2}{|c|}{$53(24,81)$} & \multicolumn{2}{|c|}{$56(27,82)$} & 0.22 \\
\hline Disease duration (months) & \multicolumn{2}{|c|}{$36(3,408)$} & \multicolumn{2}{|c|}{$30(3,408)$} & \multicolumn{2}{|c|}{$72(15,240)$} & 0.0014 \\
\hline \multicolumn{8}{|l|}{ On methotrexate } \\
\hline Yes & 70 & $87.5 \%$ & 49 & $85.96 \%$ & 21 & $91.3 \%$ & 0.72 \\
\hline No & 10 & $12.5 \%$ & 8 & $14.04 \%$ & 2 & $8.7 \%$ & \\
\hline
\end{tabular}

Data presented as $n$ (\%). Age and disease duration expressed as median (minimum, maximum) values

*Median two-sample test $p$ value

Table 2 Clinical and imaging scores at Visit 1 and Visit 2 (4 months later)

\begin{tabular}{|c|c|c|c|c|c|c|c|c|c|c|c|c|c|}
\hline & \multicolumn{4}{|c|}{ Total $(N=84)$} & \multicolumn{4}{|c|}{ Group A $(N=57)$} & \multicolumn{4}{|c|}{ Group B $\left(N=27^{*}\right)$} & \multirow{2}{*}{$\begin{array}{l}\text { Median } \\
\text { two- } \\
\text { sample } \\
\text { test } \\
p \text { value }\end{array}$} \\
\hline & $N$ & Median & Minimum & Maximum & N & Median & Minimum & Maximum & $N$ & Median & Minimum & Maximum & \\
\hline \multicolumn{14}{|l|}{ Clinical scores at Visit 1} \\
\hline Tender 28 joints & 84 & 11 & 1 & 28 & 57 & 10 & 1 & 28 & 27 & 14 & 1 & 28 & 0.050 \\
\hline Swollen 28 joints & 84 & 4 & 0 & 20 & 57 & 4 & 0 & 11 & 27 & 6 & 0 & 20 & 0.097 \\
\hline $\mathrm{HAQ}$ & 84 & 1.06 & 0 & 2.5 & 57 & 0.88 & 0 & 2.13 & 27 & 1.38 & 0.13 & 2.5 & 0.037 \\
\hline DAS28 $8_{C R P}$ & 84 & 4.5 & 3.24 & 6.64 & 57 & 4.24 & 3.24 & 6.04 & 27 & 5.15 & 3.36 & 6.64 & 0.003 \\
\hline
\end{tabular}

MRI scores at Visit 1

\begin{tabular}{|c|c|c|c|c|c|c|c|c|c|c|c|c|c|}
\hline Synovitis & 77 & 3 & 0 & 9 & 51 & 3 & 0 & 9 & 26 & 4 & 0 & 9 & 0.102 \\
\hline Tendonitis & 77 & 3 & 0 & 15 & 51 & 3 & 0 & 15 & 26 & 3 & 0 & 15 & 0.931 \\
\hline Osteitis & 77 & 2 & 0 & 34 & 51 & 2 & 0 & 34 & 26 & 3 & 0 & 24 & 0.021 \\
\hline Erosion & 76 & 5 & 0 & 50 & 50 & 4 & 0 & 50 & 26 & 9 & 1 & 47 & 0.030 \\
\hline Total inflammation & 77 & 9 & 0 & 45 & 51 & 8 & 0 & 45 & 26 & 13 & 1 & 36 & 0.050 \\
\hline
\end{tabular}

X-ray scores at Visit 1

\begin{tabular}{|c|c|c|c|c|c|c|c|c|c|c|c|c|c|}
\hline Erosions & 62 & 13 & 0 & 130 & 42 & 5.5 & 0 & 112 & 20 & 33.5 & 0 & 130 & 0.001 \\
\hline Total SvdH score & 62 & 35.5 & 0 & 217 & 42 & 20.5 & 0 & 217 & 20 & 63 & 0 & 209 & 0.031 \\
\hline \multicolumn{14}{|c|}{ Clinical scores at Visit 2} \\
\hline Swollen 28 joints & 72 & 1 & 0 & 20 & 48 & 1 & 0 & 20 & 24 & 0.5 & 0 & 9 & 0.337 \\
\hline HAQ & 72 & 0.63 & 0 & 2.13 & 48 & 0.5 & 0 & 2 & 24 & 0.75 & 0 & 2.13 & 0.155 \\
\hline
\end{tabular}

MRI scores at Visit 2

\begin{tabular}{|c|c|c|c|c|c|c|c|c|c|c|c|c|c|}
\hline Synovitis & 66 & 3 & 0 & 9 & 42 & 3 & 0 & 9 & 24 & 3 & 0 & 8 & 0.851 \\
\hline Tendonitis & 66 & 2.5 & 0 & 15 & 42 & 2 & 0 & 15 & 24 & 3 & 0 & 12 & 0.612 \\
\hline Osteitis & 68 & 2 & 0 & 34 & 44 & 2 & 0 & 32 & 24 & 3.5 & 0 & 34 & 0.102 \\
\hline Total inflammation & 66 & 9 & 1 & 53 & 42 & 9 & 1 & 43 & 24 & 9 & 1 & 53 & 1.000 \\
\hline
\end{tabular}

* Includes the four patients who transitioned from Group A to Group B on escalation of therapy (see text)

$D A S 28_{C R P}$ disease activity score (28 joints) incorporating C-reactive protein, MRI magnetic resonance imaging, SvdH Sharp van der Heijde, HAQ Health assessment questionnaire 
Table 3 Direction of change for MRI scores from Visit 1 to Visit 2

\begin{tabular}{|c|c|c|c|c|c|}
\hline & \multicolumn{2}{|c|}{ Group A } & Gro & & Tota \\
\hline & $n$ & $\%$ & $n$ & $\%$ & \\
\hline \multicolumn{6}{|c|}{ Change in MRI(i) } \\
\hline Decrease & 16 & 38.1 & 13 & 54.17 & 29 \\
\hline Increase & 16 & 38.1 & 3 & 12.5 & 19 \\
\hline No change & 10 & 23.81 & 8 & 33.33 & 18 \\
\hline Total & 42 & & 24 & & 66 \\
\hline \multicolumn{6}{|c|}{ Change in MRI osteitis } \\
\hline Decrease & 15 & 34.09 & 8 & 33.33 & 23 \\
\hline Increase & 9 & 20.45 & 6 & 25 & 15 \\
\hline No change & 20 & 45.45 & 10 & 41.67 & 30 \\
\hline Total & 44 & & 24 & & 68 \\
\hline \multicolumn{6}{|c|}{ Change in MRI synovitis } \\
\hline Decrease & 15 & 35.71 & 11 & 45.83 & 26 \\
\hline Increase & 9 & 21.43 & 1 & 4.17 & 10 \\
\hline No change & 18 & 42.86 & 12 & 50 & 30 \\
\hline Total & 42 & & 24 & & 66 \\
\hline \multicolumn{6}{|c|}{ Change in MRI tenosynovitis } \\
\hline Decrease & 12 & 28.57 & 9 & 37.5 & 21 \\
\hline Increase & 10 & 23.81 & 5 & 20.83 & 15 \\
\hline No change & 20 & 47.62 & 10 & 41.67 & 30 \\
\hline Total & 42 & & 24 & & 66 \\
\hline \multicolumn{6}{|c|}{ Change in MRI erosion } \\
\hline Increase & 3 & 6.98 & 5 & 20.83 & 8 \\
\hline No change & 40 & 93.02 & 19 & 79.17 & 59 \\
\hline Total & 43 & & 24 & & 67 \\
\hline
\end{tabular}

$M R I$ magnetic resonance imaging, MRI(i) total MRI inflammation score at the wrist (sum of synovitis, osteitis and tendonitis scores)

(20.83\%) in Group B). The majority had no change in MRI erosion scores (93.0\% and $79.17 \%$ respectively).

Combining groups, there was a weak positive correlation between $\triangle \mathrm{MRI}(\mathrm{i})$ and $\triangle \mathrm{DAS} 28_{\mathrm{CRP}}$ (Spearman's $0.36, p=0.003)$. Only $\Delta$ MRI tenosynovitis correlated with $\triangle \mathrm{DAS} 28_{\mathrm{CRP}}$ (Spearman's 0.33, $p=0.007$ ); MRI subscores for $\Delta$ osteitis, $\Delta$ synovitis and $\Delta$ erosions did not (Table 4). Multiple linear regression analysis was used to assess the effect of group and $\triangle \mathrm{DAS} 28_{\mathrm{CRP}}$ on $\triangle \mathrm{MRI}(\mathrm{i})$ adjusting for age, gender, duration, anti-CCP titre and MRI erosion score at Visit $1 . \triangle \mathrm{DAS} 28_{\mathrm{CRP}}$ was associated with $\triangle M R I(i)$ so that for every unit increase in $\triangle \mathrm{DAS} 28_{\mathrm{CRP}}$ there was a $1.77-\mathrm{U}$ increase in $\Delta \mathrm{MRI}(\mathrm{i})(p=$ 0.056). Findings are summarized in Fig. 2.

\section{Change in MRI score did not differ between groups}

There was no difference between groups for $\triangle \mathrm{MRI}(\mathrm{i})$ although the point estimate of the fall in score was
Table 4 Correlations between $\triangle \mathrm{DAS} 28_{\mathrm{CRP}}$ and $\Delta \mathrm{MRI}$ scores

\begin{tabular}{llll}
\hline $\begin{array}{l}\text { Change in MRI scores } \\
\text { (over 4 months) }\end{array}$ & $\begin{array}{l}\text { Number of } \\
\text { observations }\end{array}$ & $\begin{array}{l}\text { Spearman correlation } \\
\text { coefficients }\end{array}$ & $\begin{array}{l}p \\
\text { value }\end{array}$ \\
\hline$\Delta$ total MRI(i) & $66^{*}$ & 0.36 & 0.003 \\
$\Delta$ osteitis & 68 & 0.12 & 0.33 \\
$\Delta$ synovitis & 66 & 0.21 & 0.09 \\
$\Delta$ tendonitis & 66 & 0.33 & 0.007 \\
$\Delta$ erosion & 67 & -0.02 & 0.87 \\
\hline
\end{tabular}

*Sixty-six scan pairs available, includes eight pairs from the four patients who transitioned from Group A to Group B

$D A S 28_{C R P}$ disease activity score (28 joints) incorporating C-reactive protein, $M R I$ magnetic resonance imaging, MRI(i) total MRI inflammation score at the wrist (sum of synovitis, osteitis and tendonitis scores)

numerically greater for Group A (-3.68 (95\% CI -6.16 to -1.20$)$ ) than Group B $(-0.84$ (95\% CI -4.27 to 2.60$)$, $p=0.18)$. We did note an outlier in Group B in whom there was a significant fall in the DAS28 ${ }_{\text {CRP }}$ (from 5.15 (V1) to 3.31 (V2)) despite a marked rise in MRI inflammation scores (from 25 to 53). Data for this patient were excluded in a sensitivity analysis but the difference between groups remained non-significant $(p=0.27)$.

\section{MRI erosion score at V1 was associated with change in MRI(i)}

The MRI erosion score (V1) was negatively associated with $\triangle \mathrm{MRI}(\mathrm{i})$ (parameter estimate $-0.28 ; p=0.0052$ ), so those with higher MRI erosion scores at V1 had a greater fall in MRI(i) from V1 to V2. For every 1-U increase in the V1 erosion score, the MRI(i) score would be estimated to fall from $\mathrm{V} 1$ to $\mathrm{V} 2$ by $0.28 \mathrm{U}$. The $\mathrm{X}$-ray erosion score (V1) was not associated with $\Delta \mathrm{MRI}(\mathrm{i})(p=0.79)$.

\section{Discussion}

This is the first real-world study to investigate the link between changes in clinical disease activity and imaging (MRI) joint inflammation, in a cohort of RA patients undergoing escalation of therapy according to T2T principles. In this setting, the clinical target is most often a low disease activity state or complete remission (DAS28 $\left.8_{\text {CRP }} \leq 1.9\right)$. It has been suggested that more stringent imaging targets should be used so that patients are treated aggressively, aiming to reach a state where there is no imaging evidence of inflammation at all [19]. MRI scanning can inform about inflammation affecting different tissues including synovium (synovitis), bone (osteitis) and tendon sheaths/tendons (tenosynovitis). We found a significant but relatively weak association between change in clinical disease activity as measured by the DAS $28_{\text {CRP }}$ over the 4-month period after escalation of therapy, and change in the total MRI inflammation score. The only MRI sub-score separately associated with $\triangle \mathrm{DAS} 28_{\mathrm{CRP}}$ was the $\triangle \mathrm{MRI}$ tendonitis score, although there was a trend towards an association with 


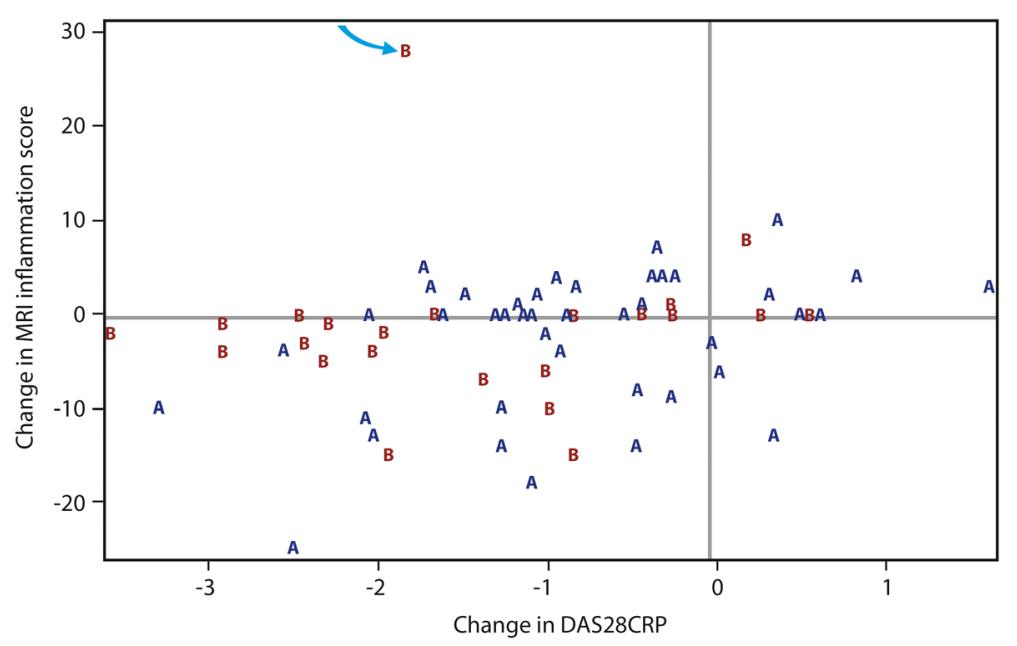

Fig. 2 Change in MRI inflammation score (over 4 months) plotted against change in DAS28 CRP for each individual patient (labelled A or B according to group). Arrow points to an outlier in Group B (see text). DAS28 $8_{C R P}$ disease activity score (28 joints) incorporating C-reactive protein, MRI magnetic resonance imaging

the $\triangle$ MRI synovitis score. These findings are consistent with observations by Krabben et al. [11], who found in their analysis of 1790 joints in 179 patients that synovitis and tenosynovitis on MRI were independently associated with clinical swelling. In that study, MRI inflammation was most often observed at the wrist, validating our choice of site for MRI scanning.

Although we did observe an association between change in clinical disease activity and change in MRI inflammation scores, it was obvious from inspection of the data that many individual patients improved clinically over the 4 months after treatment escalation, with only minor changes in their MRI inflammation scores. In some instances MRI inflammation worsened despite clinical improvement. Could this be due to the much greater sensitivity of MRI for detecting joint inflammation? Clinicians are familiar with high-grade clinical joint inflammation manifesting as Virchow's triad of redness, swelling and tenderness, while lesser degrees may only be detectable as pain on movement. MRI can detect inflammation below the clinical threshold by revealing thickened synovium and tenosynovium, which often enhance post contrast, reflecting increased vascularity. However, if MRI inflammation simply represents the low end of the inflammatory spectrum, one might expect a closer correlation between clinical and imaging observations than we have found. A ceiling effect for MRI inflammation scores could be part of the explanation. Thus, a severely inflamed wrist joint might achieve a very high MRI inflammation score, which remains high even when joint swelling and tenderness have subsided. A period of 4 months might also have been insufficient time for MRI(i) to fall in some patients [20]. Another explanation for the relative disparity between changes in clinical and MRI scores is that they capture slightly different information. Clinical disease activity scores incorporate (through joint tenderness) an assessment of pain and this is missing from the MRI inflammation score. The MRI inflammation score incorporates osteitis, which is not directly detectable clinically. Additionally, low-grade synovitis may occur in normal individuals, especially older people [21], and this could contribute to background "noise" within the MRI inflammation score. In this study we have used the DAS28 $8_{\text {CRP }}$ as a "gold standard" against which changes in MRI inflammation were compared, but this measure has its own shortcomings and in some studies the physician global assessment is a better predictor of clinical decision-making [22].

Another possible explanation for the relatively poor correlation between change in MRI inflammation scores and change in the DAS28 $8_{\text {CRP }}$ might be that MRI scans in this study imaged one region only, the dominant wrist, while the DAS28 $8_{\text {CRP }}$ reflected disease activity at 28 joints. We were able to retrieve joint count data and found evidence for tenderness and/or swelling at the scanned wrist in $71.2 \%$ of patients at Visit 1 and $47 \%$ at Visit 2. Thus, the wrist was quite frequently involved. How well do MRI scans of the wrist reflect, or in other words act as a surrogate marker for, overall rheumatoid disease activity? The evidence for this is indirect. In an early study by McQueen et al. [12], MRI wrist bone oedema (osteitis) scores were predictive of radiographic erosion progression at the hands and feet 6 years later. In 2014, Baker et al. [20] reported a very similar result for MRI osteitis and synovitis from one hand (wrist and MCPs), which were again highly predictive of radiographic progression in the joints of the hands and feet 1 and 2 years later. In 2017, Glinatsi et al. [23] published a post-hoc analysis of clinical trial data from the CIMESTRA/OPERA cohorts and concluded that MRI- 
assessed inflammation at the wrist (rather than at the MCP joints) was the most important parameter reflecting physical function, pain and global assessment of disease activity in early RA. Thus, although MRI scanning of one joint area may potentially suffer from "sampling error", there is evidence that the wrist is a particularly informative region.

One of the aims of the study was to compare MRI parameters between patients escalating from one cDMARD combination to another cDMARD combination (Group A) and patients escalating to anti-TNF therapy/cDMARDs (Group B). Group B patients, who were further up the $\mathrm{T} 2 \mathrm{~T}$ escalation ladder, differed from Group A patients in that they had higher scores for MRI erosion and osteitis at their first visit. Interestingly, there were no differences between groups in terms of synovitis or tendonitis. This is consistent with the known association between MRI osteitis and erosion progression in RA [12, 13], and emphasizes that bone inflammation (osteitis) may be qualitatively and prognostically different from soft tissue joint inflammation (synovitis and tenosynovitis). Molenaar et al. [24] observed that clinically relevant damage progression can occur in patients in prolonged remission. Thus, MRI may have an important role in the detection of osteitis and prediction of damage progression; a role that cannot be duplicated by clinical assessment alone. Very few of our patients demonstrated MRI erosion progression over 4 months but longer follow-up would be required to determine whether those with clinically normal joints but high levels of osteitis had a worse erosive outcome. We did not find evidence for a greater reduction in MRI inflammation scores in those receiving anti-TNF therapy than those on cDMARDs. Although Groups A and B cannot be directly compared because of differences in disease duration, this supports the observations of O'Dell et al. [25] who showed equivalent clinical improvement in patients treated with triple therapy and those receiving anti-TNF/ methotrexate therapy. Our findings also go some way towards validating the NZ PHARMAC escalation protocol that requires patients to have first tried cDMARD therapy before escalating to more costly bDMARD options.

What are the implications of our findings for the treating clinician? T2T regimens using clinical targets have been profoundly effective in limiting the progression of joint damage and disability in RA patients on CDMARD and bDMARD regimens [26]. If the goal posts are shifted to more stringent imaging targets, what is the evidence for better outcomes? The OMERACT group have studied this by looking for evidence of a "safe level" of MRI inflammation below which damage progression did not to occur, using MRI data from several RA clinical trial cohorts [27]. Gandjbakhch et al. identified an "acceptable MRI inflammatory activity state" as a RAMRIS synovitis score below 6 . In these individuals, radiographic progression rarely occurred. As yet there is no evidence that clinical or functional outcomes are better in such patients. Our study has indicated considerable disparity between clinical disease activity and MRI joint inflammation, and has emphasized that the goal of complete imaging resolution of inflammation is far off in the majority of real-world patients. Is it appropriate to treat these individuals more aggressively? Issues of feasibility, cost and patient acceptability mean that clinical goals are probably to be preferred over MRI goals at least in the short term. Whether ultrasound can step into the gap remains an open question [28]. Both the ARCTIC [29] and the TaSER [30] studies have recently investigated whether ultrasound imaging can improve patient outcomes if used by clinicians to inform decision-making. Both studies concluded that an ultrasound-driven T2T strategy was not associated with significantly better clinical or imaging outcomes than a DAS28-driven strategy. Further large prospective studies of this type are now needed for MRI scanning.

Our study has a number of limitations. Firstly, the study was observational. It was not possible to stipulate what disease-suppressing therapies could be given and the associations we observed could have been influenced by individual DMARDs. We considered stratifying patients according to therapy but there were many combinations of cDMARDs used and groups would have been too small for useful comparison. However, we did establish that a similar proportion of Group A and Group B patients were taking methotrexate at their first visit. Secondly, although we found no difference between groups in clinical or MRI responses to therapy, this comparison was flawed by insufficient statistical power and also by the fact that the groups differed in terms of disease duration and radiographic erosions at baseline [31]. Despite extending enrolment by a year we did not reach the numbers required to satisfy the original power calculation. Nevertheless, our comparative clinical and MRI data do shed light on what is actually happening in terms of joint inflammation in rheumatoid patients undergoing escalation of therapy in routine rheumatology practice, and should be of help to clinicians working towards the aim of improving quality of life in their patients.

\section{Conclusions}

Full clinical and MRI data were available at two time points, before and 4 months after a change of therapy, in 66 out of 80 RA patients undergoing real-world T2T escalation. There was no or minimal change in the median MRI inflammation score over 4 months, despite clinical improvement. There was no difference in the MRI inflammation response between those escalating to a different cDMARD regimen and those escalating to a cDMARD/anti-TNF regimen. For the entire group there was a significant correlation between the change in the 
DAS28 $8_{\mathrm{CRP}}$ and the change in the MRI inflammation score (Spearman's 0.36, $p=0.003$ ), but this was relatively weak. These results suggest that MRI outcomes should not be adopted to direct T2T escalation as clinically driven strategies are already successful in improving patient outcomes. A randomized controlled trial comparing MRI-driven and DAS-driven strategies is needed.

\begin{abstract}
Abbreviations
3T-MRI: 3-Tesla magnetic resonance imaging; Anti-CCP: Anti-cyclic citrullinated peptide antibodies; bDMARD: Biological disease-modifying therapy; CDMARD: Conventional disease-modifying therapy; CRP: C-reactive protein; DAS28CRP: Disease activity score (28 joints) incorporating CRP; dRUJ: Distal radioulnar joint; FS: Fat saturation; GdDTPA: Gadolinium diethylenetriamine pentaacetic acid; ICC (av): Intraclass correlation coefficient (average); MC: Metacarpal; MRI(i): Total MRI inflammation score at the wrist (sum of synovitis, osteitis and tendonitis scores); MTX/anti-TNF: Methotrexate combined with anti-tumour necrosis therapy; OMERACT: Outcome Measures in Rheumatology; PD: Proton density; PHARMAC: Pharmaceutical Management Agency (New Zealand); RA: Rheumatoid arthritis; RAMRIS: Rheumatoid Arthritis Magnetic Resonance Imaging Score; SAS: Statistical Analysis System; SPAIR: Spectral adiabatic inversion recovery; SvdH: Sharp van der Heijde; T1w: T1 weighted; T2T: Treat to target; T2w: T2 weighted; V1: Visit one; V2: Visit

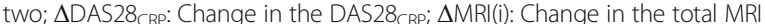
inflammation score (wrist)
\end{abstract}

\section{Acknowledgements}

The authors thank all patients who participated in this study. They thank Mrs Megan Johnston, Ms Niamh Fanning and Clinical Nurse Specialist Jan Ipenburg for their assistance with patient recruitment for the Christchurch centre. The authors also thank the staff of Specialist Radiology and MRI Greenlane (SRG, Auckland), especially Mrs Desiree Mulders, charge radiographer, for their technical expertise with MRI scanning and documentation. They thank the staff of Hagley Radiology, Christchurch, for their technical expertise in performing MRI scans. The authors thank Dr Quentin Reeves (radiologist SRG) for his assistance in developing the MRI scan protocol used in the study.

\section{Funding}

The study was supported by the Auckland Medical Research Foundation (grant reference 1114001), Arthritis New Zealand (R234) and Lotteries Health NZ (3707583)

\section{Availability of data and materials}

The datasets used and/or analysed during the present study are available from the corresponding author on reasonable request

\section{Disclosures}

FMM has no disclosures. ND reports personal fees from Abbvie, outside the submitted work. The remaining authors have no disclosures.

\section{Authors' contributions}

FMM created and designed the project, supervised collection, analysis and interpretation of the data and wrote the manuscript. PC contributed to the study design, patient enrolment, data collection and analysis plus review of the manuscript. TP contributed to MRI data management and analysis and review of the manuscript. DD supervised patient recruitment and data collection and management. AL advised on initial study design and power calculation, performed statistical analysis and advised on manuscript presentation. ND advised on study design, scored X-ray images and contributed to manuscript submission. LS assisted in patient recruitment, data collection and review and advised on analysis. KL participated in the initial study design, oversaw review by the regulatory authorities and reviewed the manuscript. AD participated in study creation, read all MRI scans and formatted MRI scan data and reviewed the manuscript. Approval of the final version of the manuscript was obtained from all co-authors.

\section{Authors' information}

Not applicable.

\section{Ethics approval and consent to participate}

The New Zealand Health and Disability Ethics Committees approved this study design (14/CEN/118/AM01. hdecs@moh.govt.nz). Written informed consent was obtained from all subjects prior to participation in study.

\section{Consent for publication}

Not applicable.

\section{Competing interests}

The authors declare that they have no competing interests.

\section{Publisher's Note}

Springer Nature remains neutral with regard to jurisdictional claims in published maps and institutional affiliations.

\section{Author details}

'Department of Molecular Medicine and Pathology, Faculty of Medical and Health Sciences, The University of Auckland, Private Bag 92019, Auckland, New Zealand. ${ }^{2}$ Department of Radiology, Auckland City Hospital, 2 Park Rd, Grafton, Auckland 1023, New Zealand. ${ }^{3}$ Section of Epidemiology \& Biostatistics, School of Population Health (Tamaki Campus), University of Auckland, Auckland 1142, New Zealand. "Bone \& Joint Research Group, Department of Medicine, The University of Auckland, Private Bag 92019, Auckland, New Zealand. ${ }^{5}$ Department of Rheumatology, Greenlane Clinical Centre, Auckland District Health Board, 214 Green Lane West, Epsom, Auckland 1051, New Zealand. ${ }^{6}$ Department of Rheumatology, Immunology \& Allergy, Christchurch Hospital, PO Box 4710, Christchurch 8140, New Zealand. ${ }^{7}$ Department of Medicine, University of Otago, PO Box 4345, Christchurch 8140, New Zealand.

Received: 31 July 2017 Accepted: 18 September 2017

Published online: 24 October 2017

\section{References}

1. Grigor C, Capell H, Stirling A, McMahon AD, Lock P, Vallance R, et al. Effect of a treatment strategy of tight control for rheumatoid arthritis (the TICORA study): a single-blind randomised controlled trial. Lancet. 2004;364(9430):263-9.

2. Smolen JS, Landewe R, Bijlsma J, Burmester G, Chatzidionysiou K, Dougados $M$, et al. EULAR recommendations for the management of rheumatoid arthritis with synthetic and biological disease-modifying antirheumatic drugs: 2016 update. Annals of the Rheumatic Diseases. 2017;76(6):960-77.

3. Pharmac. Decision relating to the funding of TNF inhibitors (Humira and Enbrel) and gabapentin (Neurontin) 2015 [updated 19 June 201720.6.17]. Available from: https:/www.pharmac.govt.nz/news/notification-2015-09-09tnf-inhibitors/.

4. van Riel PLCM, Fransen J. DAS28: a useful instrument to monitor infliximab treatment in patients with rheumatoid arthritis. Arthritis Research and Therapy. 2005;7(5):189-90.

5. Prevoo ML, Van 't Hof MA, Kuper $H H$, van Leeuwen MA, van de Putte $L B$, van Riel PL. Modified disease activity scores that include twenty-eight-joint counts. Development and validation in a prospective longitudinal study of patients with rheumatoid arthritis.[see comment]. Arthritis \& Rheumatism. 1995;38(1):44-8

6. McQueen FM, Ostergaard M. Established rheumatoid arthritis - new imaging modalities. Baillieres Best Pract Res Clin Rheumatol. 2007;21(5):841-56.

7. McQueen FM, McHaffie A, Clarke A, Lee AC, Reeves Q, Curteis B, et al. MRl osteitis predicts cartilage damage at the wrist in RA: a three-year prospective 3 T MRI study examining cartilage damage. Arthritis Research and Therapy. 2014;16:R33.

8. Ostergaard M, Peterfy C, Conaghan P, McQueen F, Bird P, Ejbjerg B, et al. OMERACT Rheumatoid Arthritis Magnetic Resonance Imaging Studies. Core set of MRI acquisitions, joint pathology definitions, and the OMERACT RAMRI scoring system. J Rheumatol. 2003;30(6):1385-6.

9. Haavardsholm EA, Ostergaard M, Ejbjerg BJ, Kvan NP, Kvien TK. Introduction of a novel magnetic resonance imaging tenosynovitis score for rheumatoid arthritis: reliability in a multireader longitudinal study. Annals of the Rheumatic Diseases. 2007:66(9):1216-20.

10. Brown AK, Quinn MA, Karim Z, Conaghan PG, Peterfy CG, Hensor E, et al. Presence of significant synovitis in rheumatoid arthritis patients with disease-modifying antirheumatic drug-induced clinical remission: evidence 
from an imaging study may explain structural progression. Arthritis. Rheumatism. 2006;54(12):3761-73.

11. Krabben A, Stomp W, Huizinga TW, van der Heijde D, Bloem JL, Reijnierse $\mathrm{M}$, et al. Concordance between inflammation at physical examination and on MRI in patients with early arthritis. Annals of the Rheumatic Diseases. 2015;74(3):506-12.

12. McQueen F, Benton N, Perry D, Crabbe J, Robinson E. Bone Edema Scored on Magnetic Resonance Imaging Scans of the Dominant Carpus at Presentation Predicts Radiographic Joint Damage of the Hands and Feet Six Years Later in Patients with Rheumatoid Arthritis. Arthritis Rheum. 2003; 48(7):1814-27

13. Hetland ML, Ejbjerg B, Horslev-Petersen $\mathrm{K}$, Jacobsen $\mathrm{S}$, Vestergaard A, Jurik $\mathrm{AG}$, et al. MRI bone oedema is the strongest predictor of subsequent radiographic progression in early rheumatoid arthritis. Results from a 2-year randomised controlled trial (CIMESTRA). Annals of the Rheumatic Diseases. 2009;68(3):384-90

14. Ostergaard M, Emery P, Conaghan PG, Fleischmann R, Hsia EC, Xu W, et al, Significant improvement in synovitis, osteitis, and bone erosion following golimumab and methotrexate combination therapy as compared with methotrexate alone: a magnetic resonance imaging study of 318 methotrexate-naive rheumatoid arthritis patients. Arthritis. Rheumatism. 2011;63(12):3712-22.

15. Conaghan PG, Peterfy C, Olech E, Kaine J, Ridley D, Dicarlo J, et al. The effects of tocilizumab on osteitis, synovitis and erosion progression in rheumatoid arthritis: results from the ACT-RAY MRI substudy. Annals of the Rheumatic Diseases. 2014;73(5):810-6.

16. Emery P, van der Heijde D, Ostergaard M, Conaghan PG, Genovese MC, Keystone EC, et al. Exploratory analyses of the association of MRI with clinical, laboratory and radiographic findings in patients with rheumatoid arthritis. Annals of the Rheumatic Diseases. 2011;70(12):2126-30.

17. McQueen FM, Clarke A, McHaffie A, Reeves Q, Williams M, Robinson E, et al. Assessment of cartilage loss at the wrist in rheumatoid arthritis using a new MRI scoring system. Annals of the Rheumatic Diseases. 2010;69:1971-5.

18. van der Heijde DM, van Riel PL, Nuver-Zwart IH, Gribnau FW. vad de Putte LB. Effects of hydroxychloroquine and sulphasalazine on progression of joint damage in rheumatoid arthritis. Lancet. 1989:1(8646):1036-8.

19. Porter D, Dale J, Sattar N. How low to aim in rheumatoid arthritis? Learning from other disciplines. Annals of the Rheumatic Diseases. 2014;73(3):480-2

20. Baker JF, Ostergaard M, Emery P, Hsia EC, Lu J, Baker DG, et al. Early MRI measures independently predict 1-year and 2-year radiographic progression in rheumatoid arthritis: secondary analysis from a large clinical trial. Annals of the Rheumatic Diseases. 2014;73(11):1968-74.

21. Nieuwenhuis WP, Mangnus L, van Steenbergen HW, Newsum EC, Huizinga TW, Reijnierse $\mathrm{M}$, et al. Older age is associated with more MRI-detected inflammation in hand and foot joints. Rheumatology (Oxford). 2016;55(12): 2212-9.

22. Lindsay K, Ibrahim G, Sokoll K, Tripathi M, Melsom RD, PS H. The composite DAS Score is impractical to use in daily practice: evidence that physicians use the objective component of the DAS in decision making. Journal of clinical rheumatology. 2009;15(5):223-5.

23. Glinatsi D, Baker JF, Hetland ML, Horslev-Petersen K, Ejbjerg BJ, StengaardPedersen $\mathrm{K}$, et al. Magnetic resonance imaging assessed inflammation in the wrist is associated with patient-reported physical impairment, global assessment of disease activity and pain in early rheumatoid arthritis: longitudinal results from two randomised controlled trials. Annals of the Rheumatic Diseases. 2017;76(10):1707-15.

24. Molenaar ET, Voskuyl AE, Dinant HJ, Bezemer PD, Boers M, Dijkmans BA. Progression of radiologic damage in patients with rheumatoid arthritis in clinical remission. Arthritis \& Rheumatism. 2004;50(1):36-42.

25. O'Dell JR, Mikuls TR, Taylor TH, Ahluwalia V, Brophy M, Warren SR, et al. Therapies for active rheumatoid arthritis after methotrexate failure. N Engl J Med. 2013;369(4):307-18

26. Steunebrink LM, Vonkeman $H E$, ten Klooster PM, Hoekstra M, van Riel PL, van de Laar MA. Recently diagnosed rheumatoid arthritis patients benefit from a treat-to-target strategy: results from the DREAM registry. Clin Rheumatol. 2016;35(3):609-15.

27. Gandjbakhch F, Haavardsholm EA, Conaghan PG, Ejbjerg B, Foltz V, Brown $A K$, et al. Determining a magnetic resonance imaging inflammatory activity acceptable state without subsequent radiographic progression in rheumatoid arthritis: results from a followup MRI study of 254 patients in clinical remission or low disease activity. J Rheumatol. 2014;41(2):398-406.
28. Mandl P, Kurucz R, Niedermayer D, Balint PV, Smolen JS. Contributions of ultrasound beyond clinical data in assessing inflammatory disease activity in rheumatoid arthritis: current insights and future prospects. Rheumatology (Oxford). 2014:53(12):2136-42.

29. Haavardsholm EA, Aga AB, Olsen IC, Lillegraven S, Hammer HB, Uhlig T, et al. Ultrasound in management of rheumatoid arthritis: ARCTIC randomised controlled strategy trial. Bmj. 2016;i4205:354.

30. Dale J, Stirling A, Zhang R, Purves D, Foley J, Sambrook M, et al. Targeting ultrasound remission in early rheumatoid arthritis: the results of the TaSER study, a randomised clinical trial. Annals of the Rheumatic Diseases. 2016; 75(6):1043-50.

31. Hyrich KL, Watson KD, Silman AJ, Symmons DP. British Society for Rheumatology Biologics R. Predictors of response to anti-TNF-alpha therapy among patients with rheumatoid arthritis: results from the British Society for Rheumatology Biologics Register. Rheumatology (Oxford). 2006;45(12):1558-65

\section{Submit your next manuscript to BioMed Central and we will help you at every step:}

- We accept pre-submission inquiries

- Our selector tool helps you to find the most relevant journal

- We provide round the clock customer support

- Convenient online submission

- Thorough peer review

- Inclusion in PubMed and all major indexing services

- Maximum visibility for your research

Submit your manuscript at www.biomedcentral.com/submit
Biomed Central 